\title{
EXPONENTIALLY STABLE STATIONARY SOLUTIONS FOR STOCHASTIC EVOLUTION EQUATIONS AND THEIR PERTURBATION
}

\author{
TOMÁS CARABALLO, PETER E. KLOEDEN, AND BJÖRN SCHMALFUSS
}

\begin{abstract}
We consider the exponential stability of stochastic evolution equations with Lipschitz continuous non-linearities when zero is not a solution for these equations. We prove the existence of a non-trivial stationary solution which is exponentially stable, where the stationary solution is generated by the composition of a random variable and the Wiener shift. We also construct stationary solutions with the stronger property of attracting bounded sets uniformly. The existence of these stationary solutions follows from the theory of random dynamical systems and their attractors. In addition, we prove some perturbation results and formulate conditions for the existence of stationary solutions for semi-linear stochastic partial differential equations with Lipschitz continuous non-linearities.
\end{abstract}

\section{Introduction}

The exponential stability of stochastic partial differential equations is an important problem, and it has received considerable attention during the recent decades as the vast literature on this topic shows. Our aim here is to study the exponential stability of non-trivial stationary solutions of these equations.

The investigation of stability for constant stationary solutions for finite dimensional stochastic differential equations goes back to Hasmínskiur [14] using Lyapunov functions for the generator of the Markov semi-group. These ideas have been extended by Mao [20] in the finite dimensional context, while non-constant stationary solutions have been treated in Schmalfuß [24]. Here we will generalize some techniques from these last two publications.

For infinite dimensional (parabolic) stochastic differential equations the problem of exponentially stable constant stationary solutions has been considered by Caraballo and Real [5] (see also [3], [4]), Liu and Mao [19], Chow [7], Haussmann [15] and Ichikawa [16] among others.

In contrast to these constant stationary solutions we will investigate the asymptotic exponential stability of non-trivial stationary solutions. In this respect, we will consider semilinear stochastic evolution equations with Lipschitz continuous non-linearities. Under suitable assumptions we prove the existence of a unique stationary solution by using a fixed point argument based on the pullback technique. This stationary solution turns to be exponentially stable in mean square, and also

Date: June, 2004.

Key words and phrases. random dynamical systems, stationary solutions, exponential stability, stabilization. 
in the almost sure sense.

Although we prove almost sure convergence to the stationary solution, the exceptional sets depend on the initial condition, so it is not possible to consider uniform convergence with respect to a bounded set of initial conditions. However, this problem will be overcome by using a technique from random dynamical systems which allows us to study the problem for exceptional sets independent of the set of initial conditions. It is unknown in general if stochastic partial differential equations with general diffusion coefficients generate random dynamical systems. However, if we suppose some kind of commutativity on these coefficients, then we are able to prove the existence of such a random dynamical system.

We prove the existence of a random fixed point, which is in fact a random variable. This random variable generates the exponentially stable stationary solution of the stochastic partial differential equation. Moreover, this stationary solution attracts bounded sets of initial conditions.

Stationary solutions in this interpretation correspond with single point random attractors, an important object in the theory of random dynamical systems, see Crauel, Debussche and Flandoli [8], Flandoli and Schmalfuß [12] or [23].

Another aim of this paper is to analyze perturbations of stochastic partial differential equations and the relation between their stationary solutions. These perturbations will be given by modifying the non-linear part of the equation. Under the assumption that the perturbations approach the non-linear part of the original equation and that the Lipschitz constants of the perturbed operators are uniformly bounded and not too large, we obtain the existence of stationary solutions which converge to the stationary solution of our original problem in the mean square sense.

For omega-wise convergence we formulate a theorem on the continuous dependence of random fixed points on a parameter.

The paper is organized as follows. In Section 2 we introduce basic concepts of stochastic evolution equations and random dynamical systems. The third section deals with the exponential stability of stationary solutions of stochastic evolution

equations in the mean square sense and almost surely. In the next section, this exponential stability is analyzed from the point of view of random dynamical systems. Then in Section 5, we consider the perturbation problems, and illustrate the results with an example in the final section.

\section{RANDOM DYNAMICAL SYSTEMS AND STOCHASTIC EVOLUTION EQUATIONS}

In this section we will describe the concept of exponentially stable stationary solutions for stochastic non-linear evolution equations generated by random fixed points. To do this we start by describing the noise driving the differential equation. Let $\left(\Omega, \mathcal{F},\left\{\mathcal{F}_{t}\right\}_{t \in \mathbb{R}}, \mathbb{P}\right)$ be a filtered probability space such that

$$
\mathcal{F}_{s} \subset \mathcal{F}_{t} \subset \mathcal{F} \quad \text { for } s \leq t .
$$

In what follows we will consider a two-sided Wiener process $W$ with values in some separable Hilbert space $U$ where the covariance $Q$ is a symmetric operator on $U$ of trace class. For instance, for the above probability space we will choose for $\Omega$ the 
set of continuous paths $C_{0}(\mathbb{R}, U)$ which are zero at zero equipped with the compact open topology. $\mathcal{F}$ is supposed to be the associated Borel- $\sigma$-algebra and $\mathbb{P}$ is defined to be the Wiener measure with respect to the covariance $Q$. For $\mathcal{F}_{t}$ we set

$$
\sigma\{\omega(u)-\omega(v): v, u \leq t\} .
$$

What we have introduced is the Brownian motion metric dynamical system which is the standard noise for random dynamical systems generated by stochastic differential equations.

Note that the above probability space is not completed. The completion of this probability space is denoted by $\left(\Omega, \overline{\mathcal{F}},\left\{\overline{\mathcal{F}}_{t}\right\}_{t \in \mathbb{R}}, \mathbb{P}\right)$ where $\left\{\overline{\mathcal{F}}_{t}\right\}_{t \in \mathbb{R}}$ has to be a normal filtration, see Da Prato and Zabczyk [10] Page 75.

We now introduce on the above non-completed probability space a measurable flow $\theta=\left\{\theta_{t}\right\}_{t \in \mathbb{R}}$ on $\Omega$ :

(1) $\quad \theta:(\mathbb{R} \times \Omega, \mathcal{F} \otimes \mathcal{B}(\mathbb{R})) \rightarrow(\Omega, \mathcal{F}), \quad \theta_{t+\tau}=\theta_{t} \circ \theta_{\tau}, t, \tau \in \mathbb{R}, \quad \theta_{0}=\operatorname{id}_{\Omega}$.

The Wiener shift operators which form the flow $\theta$

$$
\theta_{t} \omega(\cdot)=\omega(\cdot+t)-\omega(t), \quad t \in \mathbb{R}, \omega \in \Omega
$$

leave the Wiener measure $\mathbb{P}$ invariant. More precisely, $\mathbb{P}$ is ergodic with respect to $\theta$. In addition, with respect to the filtration we have that

$$
\theta_{u}^{-1} \mathcal{F}_{t}=\mathcal{F}_{t+u}
$$

for any $t, u \in \mathbb{R}$, see Arnold [1] Page 72 .

Since the above probability space is canonical we have for a Wiener process and its shift operator

$$
W(t, \omega)=\omega(t), \quad W\left(t, \theta_{s} \omega\right)=\omega(t+s)-\omega(s)=W(t+s, \omega)-W(s, \omega) .
$$

It is important to note that the measurability in $(1)$ is not true if we replace $\mathcal{F}$ by its completion, see Arnold [1] Appendix A3. However, for fixed $t$ we have the measurability of

$$
\theta_{t}:(\Omega, \overline{\mathcal{F}}) \rightarrow(\Omega, \overline{\mathcal{F}}) .
$$

Moreover, the mapping $\mathbb{R} \ni t \rightarrow \theta_{t} \omega \in C(\mathbb{R}, U)$ is continuous for a fixed $\omega \in \Omega$.

We will study the qualitative behaviour of stochastic evolution equations on some separable Hilbert space $H$ which have the form

$$
d X=A X d t+f(X) d t+B(X) d W, \quad X(0)=u_{0},
$$

where $W$ is the Wiener process on the probability space $\left(\Omega, \overline{\mathcal{F}},\left\{\overline{\mathcal{F}}_{t}\right\}_{t \in \mathbb{R}}, \mathbb{P}\right)$ introduced above. Assume that there exists a Gelfand triplet $V \subset H \subset V^{\prime}$ of separable Hilbert spaces, where $V^{\prime}$ denotes the dual of $V$ (see Temam [25] Page 55 for more details). We denote by $\|\cdot\|,\|\cdot\|_{V}$ the norms in $H$ and $V$ respectively. The inner product in $H$ will be denoted by $(\cdot, \cdot)$, and the duality mapping between $V^{\prime}$ and $V$ by $\langle\cdot, \cdot\rangle$. The random variable $u_{0}$ is supposed to be $\left(\overline{\mathcal{F}}_{0}, \mathcal{B}(H)\right)$ measurable. Let us denote by $a_{1}>0$ the constant of the injection $V \subset H$, i.e.

$$
a_{1}\|u\|^{2} \leq\|u\|_{V}^{2}, \quad \text { for } v \in V
$$


and let $-A: V \rightarrow V^{\prime}$ be a positive, linear and continuous operator for which there exists a $a_{2}<0$ such that

$$
\langle-A u, u\rangle \geq-a_{2}\|u\|_{V}^{2}, \quad \text { for all } u \in V .
$$

Then, it is well known (see, for instance, Dautray and Lions [9]) that $A$ is the generator of a strongly continuous semigroup $\{S(t)\}_{t \geq 0}$ in $H$ satisfying that

$$
\|S(t)\|_{\mathcal{L}(H)} \leq e^{a t},
$$

where $a=a_{1} a_{2}<0$.

The operator $f$ is supposed to be Lipschitz continuous from $H$ to $H$ :

$$
\left\|f\left(u_{1}\right)-f\left(u_{2}\right)\right\| \leq L_{f}\left\|u_{1}-u_{2}\right\|, \quad u_{1}, u_{2} \in H,
$$

and $B$ is supposed to be Lipschitz continuous with respect to the Hilbert-Schmidt norm $\mathcal{L}_{2}^{Q}(U, H)$ of linear operators from $U$ to $H$ :

$$
\operatorname{tr}_{H}\left(\left(B\left(u_{1}\right)-B\left(u_{2}\right)\right) Q\left(B\left(u_{1}\right)-B\left(u_{2}\right)\right)^{*}=:\left\|B\left(u_{1}\right)-B\left(u_{2}\right)\right\|_{\mathcal{L}_{2}^{Q}}^{2} \leq L_{B}\left\|u_{1}-u_{2}\right\|^{2}\right.
$$

for $u_{1}, u_{2} \in H$.

We now need the spaces $L_{2, s}:=L_{2}\left(\Omega, \overline{\mathcal{F}}_{s}, \mathbb{P} ; H\right), s \in \mathbb{R}$. We have the following theorem about the existence, uniqueness and regularity of (3).

Theorem 2.1. Suppose that $u_{0} \in L_{2,0}$. Then (3) has a unique (up to equivalence) mild solution $X(\cdot)$ on $[0, \infty)$ which has a continuous version. In addition,

$$
\mathbb{E} \int_{0}^{T}\|X(t)\|_{V}^{2} d t<\infty
$$

and

$$
\mathbb{E} \sup _{t \in[0, T]}\|X(t)\|^{2}<\infty
$$

for any $T \geq 0$.

For the existence of a mild solution see Da Prato and Zabczyk [10] Theorem 7.4. The regularity assertion can be found in Krylov and Rozovskii [17] Chapter 2.

We are interested in stationary solutions that are exponentially attracting in the $L_{2}$ sense or almost surely. Stationary solution means that the finite dimensional distributions of the solution $X$ are independent of shifts with respect to $t$. These exponentially stable stationary solutions will be generated by exponentially attracting fixed points given by an $\left(\overline{\mathcal{F}}_{0}, \mathcal{B}(H)\right)$-measurable random variable $X^{*}$ with values in $H$ such that if we choose the initial condition $u_{0}(\omega)=X^{*}(\omega)$ we have $X(t, \omega)=X^{*}\left(\theta_{t} \omega\right)$ almost surely for all $t \geq 0$, where the exceptional set may depend on $t$. This fixed point is said to be exponentially attracting if the process $(t, \omega) \rightarrow X^{*}\left(\theta_{t} \omega\right)$ (or a version of this process) attracts the solution of (3) for any (appropriate) initial condition exponentially fast in the $L_{2}$-sense or almost surely. By the $\theta_{t}$ invariance of $\mathbb{P}$ we have that

$$
\begin{aligned}
& \mathbb{P}\left(X^{*}\left(\theta_{t_{1}} \omega\right) \in B_{1}, \cdots, X^{*}\left(\theta_{t_{n}} \omega\right) \in B_{n}\right) \\
& =\mathbb{P}\left(X^{*}\left(\theta_{t_{1}+t} \omega\right) \in B_{1}, \cdots, X^{*}\left(\theta_{t_{n}+t} \omega\right) \in B_{n}\right)
\end{aligned}
$$

for $t \geq 0,0 \leq t_{1}<t_{2}<\cdots, t_{n}$ and $B_{1}, \cdots, B_{n} \in \mathcal{B}(H)$. 
Another tool that can be used to describe the stability behaviour of a stochastic evolution equation are random dynamical systems. A comprehensive presentation can be found in Arnold [1]. A random dynamical system is given by a measurable mapping

$$
\phi:\left(\mathbb{R}^{+} \times \Omega \times H, \mathcal{B}\left(\mathbb{R}^{+}\right) \otimes \mathcal{F} \otimes \mathcal{B}(H)\right) \rightarrow(H, \mathcal{B}(H)),
$$

satisfying the cocycle property:

$$
\begin{aligned}
& \phi(t+\tau, \omega, x)=\phi\left(t, \theta_{\tau} \omega, \phi(\tau, \omega, x)\right), \quad t, \tau \in \mathbb{R}^{+}, \omega \in \Omega, x \in H, \\
& \phi(0, \omega, x)=x,
\end{aligned}
$$

where $\theta$ is the flow of shift operators (Wiener shift) introduced above. We emphasize that (5) has to be satisfied for any $\omega \in \Omega$. However it is sufficient to replace $\Omega$

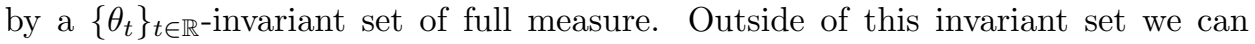
redefine $\phi$ by the identity mapping on $H$. Later on we will replace $\Omega$ by a smaller

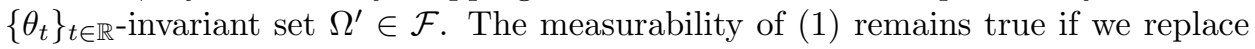
$\mathcal{F}$ by its trace $\sigma$-algebra.

The mapping $\phi$ is related to the solution of a stochastic or random differential equation. For the following we will always suppose that the mapping

$$
H \ni x \mapsto \phi(t, \omega, x) \in H
$$

is continuous for any $t, \omega$.

Although it is known that finite dimensional stochastic differential equations generate random dynamical systems (see Arnold [1] Chapter 1), this is not true in general for infinite dimensional equations. However, for particular kinds of noise we can apply the following simple lemma to obtain a random dynamical system.

Lemma 2.2. Let $\phi$ be a random dynamical system. Suppose that the mapping $T: \Omega \times H \rightarrow H$ has the following properties: For fixed $\omega \in \Omega$ the mapping $T(\omega, \cdot)$ is a homeomorphism on $H$. For fixed $x \in H$ the mappings $T(\cdot, x), T^{-1}(\cdot, x)$ are measurable. Then the mapping

$$
(t, \omega, x) \rightarrow T^{-1}\left(\theta_{t} \omega, \phi(t, \omega, T(\omega, x))\right)=: \psi(t, \omega, x)
$$

satisfies (5). Hence $\psi$ is a random dynamical system.

The measurability of $\psi$ follows because of the properties of $T$. Later on we will transform a stochastic evolution equation containing a noise term into an evolution equation without noise but with random coefficients.

A random variable $Y$ on $(\Omega, \mathcal{F}, \mathbb{P})$ with values in $H$ is called tempered if

$$
\lim _{t \rightarrow \pm \infty} \frac{\log ^{+}\left\|Y\left(\theta_{t} \omega\right)\right\|}{|t|}=0,
$$

or equivalently if $t \rightarrow\left\|Y\left(\theta_{t} \omega\right)\right\|$ has a sub-exponential growth for $t \rightarrow \pm \infty$, in other words, for $\varepsilon>0$ and $\omega \in \Omega$ there exists a $t_{0}(\varepsilon, \omega) \geq 0$ such that for $|t| \geq t_{0}(\varepsilon, \omega)$ it holds

$$
\left\|Y\left(\theta_{t} \omega\right)\right\| \leq e^{\varepsilon|t|},
$$

which means that the exponential growth rate of $t \rightarrow\left\|Y\left(\theta_{t} \omega\right)\right\|$ is zero. 
Let $\omega \rightarrow G(\omega)$ be a set valued mapping from $\Omega$ into the space of non-empty closed subsets from $H$. Such a mapping is called a random set if for any $y \in H$ the mapping

$$
\omega \rightarrow \inf _{x \in G(\omega)}\|x-y\|
$$

is a random variable. If $G$ is a random set then there exists a random variable $g$ with $g(\omega) \in G(\omega)$ (see Castaing and Valadier [6] Chapter 3). A random set $G$ is called tempered if the random variable

$$
\omega \rightarrow \sup _{x \in G(\omega)}\|x\|
$$

is tempered. It is easily seen that the set of $\omega$ for which (7), (8) are satisfied is $\left\{\theta_{t}\right\}_{t \in \mathbb{T}}$-invariant.

An $(\mathcal{F}, \mathcal{B}(H))$-measurable random variable $X^{*}$ is called a random fixed point in the sense of random dynamical systems if

$$
\phi\left(t, \omega, X^{*}(\omega)\right)=X^{*}\left(\theta_{t} \omega\right)
$$

for $t>0$, where $\omega$ is contained in a $\left\{\theta_{t}\right\}_{t \in \mathbb{T}}$-invariant set of full measure. $X^{*}$ is an exponentially stable random fixed point with respect to a closed random set $G$ containing $X^{*}$ if for any random variable $g \in G$ we have

$$
\lim _{t \rightarrow \infty}\left\|\phi(t, \omega, g(\omega))-X^{*}\left(\theta_{t} \omega\right)\right\|=0
$$

for all $\omega$ in the above $\left\{\theta_{t}\right\}_{t \in \mathbb{T}}$-invariant set of full measure with exponential speed, such that the exceptional set is independent of $t$. If $\phi$ is defined by the solution mapping of a stochastic/random differential equation then $(t, \omega) \mapsto X^{*}\left(\theta_{t} \omega\right)$ is a stationary solution of a stochastic/random differential equation.

Our strategy will be to prove that, under certain assumptions, a random dynamical system possesses a random attractor which is a single (random) point. The following definition can be found in Flandoli and Schmalfuß [12].

Definition 2.3. Let $\mathcal{D}$ be the set of all closed tempered random sets in $H$. A compact random set $\mathcal{A} \in \mathcal{D}$ is called a random attractor if the invariance property

$$
\phi(t, \omega, \mathcal{A}(\omega))=\mathcal{A}\left(\theta_{t} \omega\right)
$$

is satisfied for $\omega \in \Omega, t \geq 0$ and if, in addition, the pullback convergence

$$
\lim _{t \rightarrow \infty} \operatorname{dist}_{H}\left(\phi\left(t, \theta_{-t} \omega, D\left(\theta_{-t} \omega\right), \mathcal{A}(\omega)\right)\right)=0
$$

holds for $D \in \mathcal{D}$ and $\omega \in \Omega$.

We note that from this convergence it follows

$$
\text { (l.i.p.) } \lim _{t \rightarrow \infty} \operatorname{dist}_{H}\left(\phi\left(t, \omega, D(\omega), \mathcal{A}\left(\theta_{t} \omega\right)\right)=0,\right.
$$

where by (l.i.p.) we denote limit in probability. However, in general it does not imply $\omega$ wise almost sure convergence. Sufficient conditions for the existence of a random attractor can be found in [12].

Theorem 2.4. Suppose that the mapping $x \rightarrow \phi(t, \omega, x)$ is continuous for $t \geq 0$, and completely continuous for $t>0$ (which means that the image of every bounded 
set by the mapping $x \rightarrow \phi(t, \omega, x)$ is relatively compact). In addition, suppose there is a $G \in \mathcal{D}$ such that for any $D \in \mathcal{D}$ and $\omega \in \Omega$ there exists $T(D, \omega)>0$ such that

$$
\phi\left(t, \theta_{-t} \omega, D\left(\theta_{-t} \omega\right)\right) \subset G(\omega), \quad \text { for all } t \geq T(D, \omega) .
$$

Then there exists a unique random attractor $\mathcal{A}($ in $\mathcal{D})$.

If the random attractor $\mathcal{A}(\omega), \omega \in \Omega$, consists of a single point then $\mathcal{A}$ defines a random fixed point which attracts tempered random sets.

\section{Exponential Stability Stochastic EVOLUtion EQUATIONS}

In this section we will prove the existence of exponentially stable (both in the mean square sense and almost surely) non-trivial stationary solutions to our stochastic semi-linear partial differential equation (3). The exponential stability of trivial stationary solutions (in particular, the null solution) of stochastic PDEs has been extensively analyzed (see, for instance, $[4],[19],[16], \ldots$ and the literature cited therein). However, when zero is not a solution to the equation, it is interesting to find out whether or not there exist other stationary solutions, which are generated by random variables chosen as initial values in our problem, and to analyze their stability properties. This fact, can also be considered as a connection between the classical method for the local analysis of the long-time behaviour of stochastic partial differential equations and the global one provided by the theory of random dynamical systems.

In the sequel we consider the process $\theta_{s} W(\cdot, \omega)=W\left(\cdot, \theta_{s} \omega\right)=W(\cdot+s, \omega)-W(s, \omega)$, for $s \in \mathbb{R}$, which is also a Wiener process with covariance $Q$. For $t \geq 0$ this process is adapted to the filtration $\left\{\overline{\mathcal{F}}_{s+t}\right\}_{t \geq 0}$ which follows from (2). We will denote by $\Phi\left(\cdot, s, u_{0}\right)$ the solution of $(3)$, corresponding to the initial value $u_{0} \in L_{2, s}$, which is driven by $\theta_{s} W$, and satisfying the assertions of Theorem 2.1.

The following equality holds for $u_{0} \in L_{2,0}$ :

$$
\Phi\left(\cdot, 0, u_{0}\right)\left(\theta_{s} \cdot\right)=\Phi\left(\cdot, s, u_{s}\right)(\cdot), \quad \text { almost surely }
$$

where $u_{s}(\cdot):=u_{0}\left(\theta_{s} \cdot\right)$. Both sides of (12) are driven by the same Wiener process and the same initial condition and the fact that solutions of (3) are unique. Indeed, by $(2) u_{0}\left(\theta_{s} \cdot\right)$ is $\overline{\mathcal{F}}_{s}$-measurable.

Lemma 3.1. i) For $s \in \mathbb{R}, \tau \geq 0, u_{0} \in L_{2, s}$

$$
\Phi\left(\cdot, s+\tau, \Phi\left(\tau, s, u_{0}\right)\right)=\Phi\left(\cdot+\tau, s, u_{0}\right), \quad \text { almost surely } .
$$

ii) Set

$$
\mu:=2 a+2 L_{f}+L_{B} .
$$

Then

$$
\mathbb{E}\left\|\Phi\left(t, s, u_{0}^{1}\right)-\Phi\left(t, s, u_{0}^{2}\right)\right\|^{2} \leq e^{\mu t} \mathbb{E}\left\|u_{0}^{1}-u_{0}^{2}\right\|^{2}
$$

for $t \geq 0, s \in \mathbb{R}, u_{0}^{1}, u_{0}^{2} \in L_{2, s}$.

Proof. i) Consider the process

$$
Y(r, \omega)=\left\{\begin{array}{lrr}
\Phi\left(r, s, u_{0}\right)(\omega) & : & 0 \leq r \leq \tau \\
\Phi\left(r-\tau, s+\tau, \Phi\left(\tau, s, u_{0}\right)\right)(\omega) & : & r>\tau
\end{array},\right.
$$


which is continuous (almost surely) by the continuity of $\Phi$. For $r>\tau$ we have

$$
\begin{aligned}
Y(r)= & S(r-\tau) \Phi\left(\tau, s, u_{0}\right) \\
& +\int_{0}^{r-\tau} S(r-\tau-q) f\left(\Phi\left(q, s+\tau, \Phi\left(\tau, s, u_{0}\right)\right)\right) d q \\
& +\int_{0}^{r-\tau} S(r-\tau-q) B\left(\Phi\left(q, s+\tau, \Phi\left(\tau, s, u_{0}\right)\right) d(W(q+s+\tau)-W(s+\tau))\right. \\
= & S(r) u_{0} \\
& +\int_{\tau}^{r} S(r-q) f(Y(q)) d q+S(r-\tau) \int_{0}^{\tau} S(\tau-q) f(Y(q)) d q \\
& +\int_{\tau}^{r} S(r-q) B(Y(q)) d(W(q+s)-W(s)) \\
& +S(r-\tau) \int_{0}^{\tau} S(\tau-q) B(Y(q)) d(W(q+s)-W(s)) .
\end{aligned}
$$

The first conclusion follows if we concatenate the integrals and use the fact that for the increments of the Wiener process we have

$$
\begin{aligned}
& W\left(q_{1}+s+\tau\right)-W(s+\tau)-\left(W\left(q_{2}+s+\tau\right)-W(s+\tau)\right) \\
& \quad=W\left(q_{1}+s+\tau\right)-W(s)-\left(W\left(q_{2}+s+\tau\right)-W(s)\right) .
\end{aligned}
$$

ii) It is not hard to see by the properties of the coefficients that (3) has the trajectories in $L_{2}(0, T ; V)$ (see Krylov and Rozovskii [17], or Grecksch and Tudor [13]) which allows us to apply the Ito formula for the process $e^{-\mu t}\left\|X_{1}(t)-X_{2}(t)\right\|^{2}$ where we have denoted $X_{i}(t):=\Phi\left(t, s, u_{0}^{i}\right), i=1,2$. Thus

$$
\begin{aligned}
e^{-\mu t} & \left\|X_{1}(t)-X_{2}(t)\right\|^{2} \\
= & \left\|u_{0}^{1}-u_{0}^{2}\right\|^{2}-\mu \int_{0}^{t} e^{-\mu q}\left\|X_{1}(q)-X_{2}(q)\right\|^{2} d q \\
& +2 \int_{0}^{t} e^{-\mu q}\left\langle A\left(X_{1}(q)-X_{2}(q), X_{1}(q)-X_{2}(q)\right\rangle d q\right. \\
& +2 \int_{0}^{t} e^{-\mu q}\left(f\left(X_{1}(q)\right)-f\left(X_{2}(q)\right), X_{1}(q)-X_{2}(q)\right) d q \\
& +\int_{0}^{t} e^{-\mu q}\left\|B\left(X_{1}(q)\right)-B\left(X_{2}(q)\right)\right\|_{\mathcal{L}_{2}^{Q}}^{2} d q \\
& +2 \int_{0}^{t} e^{-\mu q}\left(X_{1}(q)-X_{2}(q),\left(B\left(X_{1}(q)\right)-B\left(X_{2}(q)\right)\right) d W\left(q, \theta_{s} \omega\right)\right) \\
\leq & \left\|u_{0}^{1}-u_{0}^{2}\right\|^{2}+\int_{0}^{t} e^{-\mu q}\left(-\mu+2 a+2 L_{f}+L_{B}\right)\left\|X_{1}(q)-X_{2}(q)\right\|^{2} d q \\
& +2 \int_{0}^{t} e^{-\mu q}\left(X_{1}(q)-X_{2}(q),\left(B\left(X_{1}(q)\right)-B\left(X_{2}(q)\right)\right) d W\left(q, \theta_{s} \omega\right)\right) . \\
\leq & \left\|u_{0}^{1}-u_{0}^{2}\right\|^{2}+2 \int_{0}^{t} e^{-\mu q}\left(X_{1}(q)-X_{2}(q),\left(B\left(X_{1}(q)\right)-B\left(X_{2}(q)\right)\right) d W\left(q, \theta_{s} \omega\right)\right) .
\end{aligned}
$$


The result follows easily by calculating the expectation if we replace $t$ by $t \wedge T_{N}$, where $T_{N}$ is a family of stopping times

$$
T_{N}(\omega)=\inf \left\{t \geq 0:\left\|X_{1}(t, \omega)\right\|^{2}+\left\|X_{2}(t, \omega)\right\|^{2} \geq N\right\}
$$

such that

$$
\lim _{N \rightarrow \infty}\left(T_{N} \wedge t\right)=t, \quad \text { almost surely, }
$$

since $X_{1}, X_{2}$ have continuous paths.

Corollary 3.2. $\Phi(t, s, \cdot)$ maps $L_{2, s}$ into $L_{2, s+t}$ continuously.

We shall now show the existence of an exponentially stable solution for (3).

Theorem 3.3. Suppose that the constant $\mu$ appearing in (13) is negative. Then there exists an exponentially attracting fixed point $X^{*} \in L_{2,0}$ generating an exponentially stable stationary solution for (3). In particular, the process $(t, \omega) \rightarrow X^{*}\left(\theta_{t} \omega\right)$ has a continuous version given by $\Phi\left(\cdot, 0, X^{*}\right)$.

Proof. We show that $\left(\Phi\left(k,-k, u_{0}\left(\theta_{-k} \cdot\right)\right)\right)_{k \in \mathbb{N}}$ is a Cauchy sequence in $L_{2,0}$ for $u_{0} \in$ $L_{2,0}$. Notice that for this $u_{0}$ we have that $u_{0}\left(\theta_{-k^{*}}\right) \in L_{2,-k}$. Indeed,

$$
\begin{aligned}
\mathbb{E} \| \Phi(k, & \left.-k, u_{0}\left(\theta_{-k} \cdot\right)\right)-\Phi\left(k-1,1-k, u_{0}\left(\theta_{1-k} \cdot\right)\right) \|^{2} \\
& =\mathbb{E}\left\|\Phi\left(k-1,1-k, \Phi\left(1,-k, u_{0}\left(\theta_{-k} \cdot\right)\right)\right)-\Phi\left(k-1,1-k, u_{0}\left(\theta_{1-k} \cdot\right)\right)\right\|^{2} \\
& \leq e^{\mu(k-1)} \mathbb{E}\left\|\Phi\left(1,-k, u_{0}\left(\theta_{-k} \cdot\right)\right)-u_{0}\left(\theta_{1-k} \cdot\right)\right\|^{2} \\
& =e^{\mu(k-1)} \mathbb{E}\left\|\Phi\left(1,0, u_{0}(\cdot)\right)-u_{0}\left(\theta_{1} \cdot\right)\right\|^{2}
\end{aligned}
$$

Here we have applied Lemma $3.1 \mathrm{i}$ ), the $\left\{\theta_{t}\right\}_{t \in \mathbb{R}}$-invariance of $\mathbb{P}$ and (12). The Cauchy sequence property follows since $\mu<0$. Let the limit of this sequence be denoted by $X^{*} \in L_{2,0}$.

$X^{*}$ as an element in $L_{2,0}$ is independent of the choice of $u_{0} \in L_{2,0}$. Indeed, for $v_{0} \in L_{2,0}$ we have that

$$
\begin{aligned}
& \mathbb{E}\left\|\Phi\left(k,-k, u_{0}\left(\theta_{-k} \cdot\right)\right)-\Phi\left(k,-k, v_{0}\left(\theta_{-k} \cdot\right)\right)\right\|^{2} \\
& \quad=\mathbb{E}\left\|\Phi\left(k, 0, u_{0}(\cdot)\right)-\Phi\left(k, 0, v_{0}(\cdot)\right)\right\|^{2} \leq e^{\mu k} \mathbb{E}\left\|u_{0}-v_{0}\right\|^{2},
\end{aligned}
$$

which goes to zero for $k \rightarrow \infty$ such that the limit of the above Cauchy sequence in $L_{2,0}$ is independent of $u_{0}$. Since $X^{*} \in L_{2,0}$ the process $\Phi\left(\cdot, 0, X^{*}\right)$ satisfies all of the conclusions of Theorem 2.1. We now show that

$$
\Phi\left(t, 0, X^{*}\right)(\cdot)=X^{*}\left(\theta_{t} \cdot\right) \quad \text { almost surely } \quad \text { for any } t \in \mathbb{R}^{+} \text {. }
$$

By the definition of $X^{*}(\omega)$ the random variable $X^{*}\left(\theta_{t} \cdot\right)$ is given by

$$
\left(L_{2}\right) \lim _{k \rightarrow \infty} \Phi\left(k,-k, u_{0}\left(\theta_{-k} \cdot\right)\right)\left(\theta_{t} \omega\right)
$$

which is equal to

$$
\left(L_{2}\right) \lim _{k \rightarrow \infty} \Phi\left(k, t-k, u_{0}\left(\theta_{t-k} \cdot\right)\right)(\omega)
$$


by (12). On account of Corollary 3.2 and Lemma 3.1 we have

$$
\begin{aligned}
\mathbb{E} \| \Phi(t, & \left.0, X^{*}\right)-X^{*}\left(\theta_{t} \cdot\right) \|^{2} \\
& =\mathbb{E} \| \Phi\left(t, 0,\left(L_{2}\right) \lim _{k \rightarrow \infty} \Phi\left(k,-k, u_{0}\left(\theta_{-k} \cdot\right)\right)-\left(L_{2}\right) \lim _{k \rightarrow \infty} \Phi\left(k, t-k, u_{0}\left(\theta_{t-k} \cdot\right)\right) \|^{2}\right. \\
& =\lim _{k \rightarrow \infty} \mathbb{E} \| \Phi\left(t, 0, \Phi\left(k,-k, u_{0}\left(\theta_{-k} \cdot\right)\right)-\Phi\left(k, t-k, u_{0}\left(\theta_{t-k} \cdot\right)\right) \|^{2}\right. \\
& =\lim _{k \rightarrow \infty} \mathbb{E} \| \Phi\left(k, t-k, \Phi\left(t,-k, u_{0}\left(\theta_{-k} \cdot\right)\right)-\Phi\left(k, t-k, u_{0}\left(\theta_{t-k} \cdot\right)\right) \|^{2}\right. \\
& \leq \lim _{k \rightarrow \infty} e^{\mu k} \mathbb{E}\left\|\Phi\left(t,-k, u_{0}\left(\theta_{-k} \cdot\right)\right)-u_{0}\left(\theta_{t-k} \cdot\right)\right\|^{2} \\
& =\lim _{k \rightarrow \infty} e^{\mu k} \mathbb{E}\left\|\Phi\left(t, 0, u_{0}\right)-u_{0}\left(\theta_{t} \cdot\right)\right\|^{2}=0,
\end{aligned}
$$

since $\mu<0$ and $t$ is fixed.

Remark 3.4. It follows from the second part of Lemma 3.1 that $\Phi\left(t, 0, u_{0}\right)-$ $\Phi\left(t, 0, X^{*}\right)$ tends to zero exponentially fast in $L_{2}(\Omega, \overline{\mathcal{F}}, \mathbb{P})$ for $t \rightarrow \infty$.

In contrast to the $L_{2}$-convergence to the stationary solution we now show that

$$
\lim _{t \rightarrow \infty}\left\|\Phi\left(t, 0, u_{0}\right)-\Phi\left(t, 0, X^{*}\right)\right\|^{2}=0, \quad \text { almost surely, }
$$

for an $\left(\overline{\mathcal{F}}_{0}, \mathcal{B}(H)\right)$ random variable $u_{0}$ and $X^{*}$ is given by the random fixed point of (3).

Theorem 3.5. Assume $\mu<0$. For a random variable $u_{0} \in L_{2,0}$ we have that

$$
\lim _{t \rightarrow \infty}\left\|\Phi\left(t, 0, u_{0}\right)-\Phi\left(t, 0, X^{*}\right)\right\|=0
$$

almost surely exponentially fast.

Proof. Take an $\left(\overline{\mathcal{F}}_{0}, \mathcal{B}(H)\right)$-measurable random variable $u_{0} \in L_{2,0}$ and let us denote

$$
\Phi\left(t, 0, u_{0}\right)=X_{1}(t), \quad \Phi\left(t, 0, X^{*}\right)=X_{2}(t) .
$$

Recall that from Lemma 3.1 it holds that

$$
\mathbb{E}\left\|X_{1}(t)-X_{2}(t)\right\|^{2} \leq e^{\mu t} \mathbb{E}\left\|u_{0}-X^{*}\right\|^{2} \quad \text { for all } t \geq 0 \quad(\mu<0) .
$$

We will first prove that there exists a positive constant $C$ such that

$$
\mathbb{E} \sup _{t \in[N, N+1]}\left\|X_{1}(t)-X_{2}(t)\right\|^{2} \leq C e^{\mu N} \mathbb{E}\left\|u_{0}-X^{*}\right\|^{2}
$$

for any $N \in \mathbb{N}$. Similarly to (14) we obtain for $t \geq N$

$$
\mathbb{E}\left\|X_{1}(t)-X_{2}(t)\right\|^{2}=\mathbb{E}\left\|X_{1}(N)-X_{2}(N)\right\| e^{\mu(t-N)} \leq \mathbb{E}\left\|u_{0}-X^{*}\right\|^{2} e^{\mu t} .
$$

Using Ito's formula for $\left\|X_{1}(t)-X_{2}(t)\right\|^{2}$ (see [17]) and taking into account that $\mu$ defined in (13) is negative, we find

$$
\begin{aligned}
& \mathbb{E} \sup _{t \in[N, N+1]}\left\|X_{1}(t)-X_{2}(t)\right\|^{2} \leq \mathbb{E}\left\|X_{1}(N)-X_{2}(N)\right\|^{2} \\
& \quad+2 \mathbb{E} \sup _{t \in[N, N+1]}\left|\int_{N}^{t}\left(X_{1}-X_{2},\left(B\left(X_{1}\right)-B\left(X_{2}\right)\right) d W(s)\right)\right| .
\end{aligned}
$$


Now we evaluate the last term in (18) by applying the Burkholder-Davis-Gundy lemma. Indeed, it follows by (17) that

$$
\begin{aligned}
& 2 \mathbb{E} \sup _{t \in[N, N+1]}\left|\int_{N}^{t}\left(X_{1}-X_{2},\left(B\left(X_{1}\right)-B\left(X_{2}\right)\right) d W(s)\right)\right| \\
& \leq C_{1} \mathbb{E}\left(\int_{N}^{N+1}\left\|X_{1}-X_{2}\right\|^{2}\left\|B\left(X_{1}\right)-B\left(X_{2}\right)\right\|_{\mathcal{L}_{2}^{Q}}^{2} d s\right)^{1 / 2} \\
& \leq C_{1} \mathbb{E}\left(\sup _{t \in[N, N+1]}\left\|X_{1}(t)-X_{2}(t)\right\|^{2} \int_{N}^{N+1}\left\|B\left(X_{1}\right)-B\left(X_{2}\right)\right\|_{\mathcal{L}_{2}^{Q}}^{2} d s\right)^{1 / 2} \\
& \leq \frac{1}{2} \mathbb{E} \sup _{t \in[N, N+1]}\left\|X_{1}(t)-X_{2}(t)\right\|^{2}+\frac{C_{1}^{2} L_{B}}{2} \mathbb{E}\left\|u_{0}-X^{*}\right\|^{2} \int_{N}^{N+1} e^{\mu s} d s \\
& \leq \frac{1}{2} \mathbb{E} \sup _{t \in[N, N+1]}\left\|X_{1}(t)-X_{2}(t)\right\|^{2}+\frac{C_{1}^{2} L_{B}}{2} e^{\mu N} \mathbb{E}\left\|u_{0}-X^{*}\right\|^{2} .
\end{aligned}
$$

Consequently, (15),(18) and (19) imply (16). The expectation of the sup-term on the right hand side just exists by Theorem 2.1. On account of the Tschebyshev inequality we have for a small $\varepsilon>0$

$$
\begin{aligned}
& \mathbb{P}\left(\sup _{t \in[N, N+1]}\left\|X_{1}(t)-X_{2}(t)\right\|^{2} \geq e^{(\mu+\varepsilon) N}\right) \\
& \quad \leq \frac{e^{-\mu N} \mathbb{E} \sup _{t \in[N, N+1]}\left\|X_{1}(t)-X_{2}(t)\right\|^{2}}{e^{\varepsilon N}} \leq \frac{C \mathbb{E}\left\|u_{0}-X^{*}\right\|^{2}}{e^{\varepsilon N}} .
\end{aligned}
$$

Note that the series $\sum_{N=1}^{\infty} e^{-\varepsilon N}$ is finite. Hence the conclusion follows by the Borel-Cantelli Lemma.

Remark 3.6. If $f(0)=B(0)=0$ then $X^{*} \equiv 0$.

\section{Exponential STABILITy FOR STOCHASTIC EVOLUTION EQUATIONS GENERATING RANDOM DYNAMICAL SYSTEMS}

The disadvantage of the method in the last section is that the exceptional sets for the definition of the random fixed point $X^{*}$ depend explicitly on the initial condition $u_{0}$ which appears in the defining Cauchy sequence. It is rather complicated to obtain the exponential convergence to the stationary solution uniformly with respect to a bounded set of initial conditions. We will try to overcome this disadvantage in this section. For this purpose it is more appropriate to consider stochastic evolution equations in the Stratonovich form. We also suppose that the diffusion part of this equation is given by

$$
B(X) \circ d W=B_{1} X \circ d w_{1}+\cdots+B_{N} X \circ d w_{N},
$$

where $w_{1}, \cdots, w_{N}$ are one-dimensional mutually independent standard Wiener processes and $W=\left(w_{1}, \cdots, w_{N}\right)$ so that the phase space $U$ for the Wiener process is given by $\mathbb{R}^{N}$, and $B_{i} \in \mathcal{L}(H)$ for $i=1, \cdots, N$. We will denote $b_{i}=\left\|B_{i}\right\|_{\mathcal{L}(H)}$. This means that we will consider the equation

$$
d X=(A X+f(X)) d t+\sum_{i=1}^{N} B_{i} X \circ d w_{i} .
$$


As our approach in this section is based on dynamical techniques, we use here the Stratonovich interpretation of our stochastic partial differential equation. Notice that, due to the equivalence which exists between the Stratonovich and the Ito interpretation for the stochastic integral, one can formulate the corresponding results for our initial problem (3) by simply redefining in a suitable way the operators in the model.

The operators $B_{i}$ generate $C_{0}$-groups which we will denote by $S_{B_{i}}$. In addition, we suppose the operators $A, B_{1}, \cdots, B_{N}$ mutually commute, what implies that these groups and the semigroup generated by $A$ are also mutually commuting. $A$ has been introduced in Section 2.

For what follows, we will fix a one-dimensional Wiener process with $U=\mathbb{R}$ and $\mathbb{E}|w(1)|^{2}=1$. We consider the one-dimensional stochastic differential equation

$$
d z=-\lambda z d t+d w(t)
$$

for some $\lambda>0$. This equation has a random fixed point in the sense of random dynamical systems generating a stationary solution known as the stationary Ornstein-Uhlenbeck process.

Lemma 4.1. Let $\lambda$ be a positive number and consider the probability space as in


$C_{0}(\mathbb{R}, \mathbb{R})$ of full measure such that

$$
\lim _{t \rightarrow \pm \infty} \frac{|\omega(t)|}{t}=0 \quad \text { for } \omega \in \bar{\Omega},
$$

and, for such $\omega$, the random variable given by

$$
z^{*}(\omega):=-\lambda \int_{-\infty}^{0} e^{\lambda \tau} \omega(\tau) d \tau
$$

is well defined. Moreover, for $\omega \in \bar{\Omega}$, the mapping

$$
\begin{aligned}
(t, \omega) \rightarrow z^{*}\left(\theta_{t} \omega\right) & =-\lambda \int_{-\infty}^{0} e^{\lambda \tau} \theta_{t} \omega(\tau) d \tau \\
& =-\lambda \int_{-\infty}^{0} e^{\lambda \tau} \omega(t+\tau) d \tau+\omega(t)
\end{aligned}
$$

is a stationary solution of (21) with continuous trajectories. In addition, for $\omega \in \bar{\Omega}$

$$
\begin{aligned}
& \lim _{t \rightarrow \pm \infty} \frac{\left|z^{*}\left(\theta_{t} \omega\right)\right|}{|t|}=0, \quad \lim _{t \rightarrow \pm \infty} \frac{1}{t} \int_{0}^{t} z^{*}\left(\theta_{\tau} \omega\right) d \tau=0 \\
& \lim _{t \rightarrow \pm \infty} \frac{1}{t} \int_{0}^{t}\left|z^{*}\left(\theta_{\tau} \omega\right)\right| d \tau=\mathbb{E}\left|z^{*}\right|<\infty
\end{aligned}
$$

Proof. The first statement follows from the law of iterated logarithm. It is easily seen that the process

$$
(\omega, t) \rightarrow \int_{-\infty}^{t} e^{-\lambda(t-\tau)} d w(\tau, \omega)
$$

is a solution of the differential equation (21). Starting from this fact we construct $z^{*}(\omega)$. By the integration by parts formula (see Øksendal, [22]) we see that (22) is 
a version of this solution. Indeed, for $w=w\left(\cdot, \theta_{-t} \omega\right)=\theta_{-t} \omega(\cdot)$ we have

$$
\begin{aligned}
\int_{0}^{t} e^{-\lambda(t-\tau)} d w\left(\tau, \theta_{-t} \omega\right)= & e^{-\lambda \cdot 0} w\left(t, \theta_{-t} \omega\right)-e^{-\lambda t} w\left(0, \theta_{-t} \omega\right) \\
& -\lambda \int_{0}^{t} e^{-\lambda(t-\tau)}(w(\tau-t, \omega)-w(-t, \omega)) d \tau \\
= & -w(-t, \omega)-\lambda \int_{0}^{t} e^{-\lambda(t-\tau)} w(t-\tau, \omega) d \tau \\
& +w(-t, \omega) e^{-\lambda t}\left(e^{\lambda t}-1\right) .
\end{aligned}
$$

Using the integral transformation $\tau \rightarrow \tau-t$ and letting $t \rightarrow \infty$ we have that the right hand side tends to $z^{*}(\omega)$ for those $\omega$ satisfying the above growth condition. Now we use that $\omega(t)=w(t, \omega)$ since we have a canonical probability space.

The stationarity of this solution follows by the invariance of the Wiener measure $\mathbb{P}$ with respect to the flow $\theta$.

The other statements follow by the ergodic theorem and the Burkholder inequality, which allows us to conclude that $\mathbb{E} \sup _{t \in[0,1]}\left|z^{*}\left(\theta_{t} \omega\right)\right|<\infty$. Together with the ergodic theorem we then obtain the first formula in $(23)$. Note that $\bar{\Omega} \in \mathcal{F}$. This follows from the fact that by the continuity of $t \rightarrow z^{*}\left(\theta_{t} \omega\right)$ the mapping $\omega \rightarrow$ $\sup _{t \in[0,1]}\left|z^{*}\left(\theta_{t} \omega\right)\right|$ is an $(\mathcal{F}, \mathcal{B}(\mathbb{R}))$-measurable mapping.

Remark 4.2. We now consider $\theta$ defined in (1) on $\bar{\Omega}$ instead of $\Omega$. This mapping has the same properties as the original one if we choose for $\mathcal{F}$ the trace $\sigma$-algebra with respect to $\bar{\Omega}$ denoted also by $\mathcal{F}$.

Let $\lambda_{1}, \cdots, \lambda_{N}$ be a set of positive numbers. For any pair $\lambda_{j}, w_{j}$ we have a stationary Ornstein-Uhlenbeck process generated by a random variable $z_{j}^{*}(\omega)$ on $\bar{\Omega}_{j}$ with properties formulated in Lemma 4.1 defined on the metric dynamical system $\left(\bar{\Omega}_{j}, \mathcal{F}_{j}, \mathbb{P}_{j}, \theta\right)$. We set

$$
(\Omega, \mathcal{F}, \mathbb{P}, \theta)
$$

where

$$
\Omega=\bar{\Omega}_{1} \times \cdots \times \bar{\Omega}_{N}, \quad \mathcal{F}=\bigotimes_{i=1}^{N} \mathcal{F}_{i}, \quad \mathbb{P}=\mathbb{P}_{1} \times \mathbb{P}_{2} \times \cdots \times \mathbb{P}_{N},
$$

and $\theta$ is the flow of Wiener shifts.

To find random fixed points for (20) we will transform this equation into an evolution equation with random coefficients but without white noise. Let

$$
T(\omega):=S_{B_{1}}\left(z_{1}^{*}(\omega)\right) \circ \cdots \circ S_{B_{N}}\left(z_{N}^{*}(\omega)\right)
$$

be a family of random linear homeomorphisms on $H$. The inverse operator is well defined by

$$
T^{-1}(\omega):=S_{B_{N}}\left(-z_{N}^{*}(\omega)\right) \circ \cdots \circ S_{B_{1}}\left(-z_{1}^{*}(\omega)\right)
$$

Because of the estimate

$$
\left\|T^{-1}(\omega)\right\| \leq e^{\left\|B_{1}\right\|\left|z_{1}^{*}(\omega)\right|} \cdot \ldots \cdot e^{\left\|B_{N}\right\|\left|z_{N}^{*}(\omega)\right|}
$$

and (23), it follows that $\left\|T^{-1}\left(\theta_{t} \omega\right)\right\|$ has sub-exponential growth as $t \rightarrow \pm \infty$ for any $\omega \in \Omega$. Hence $\left\|T^{-1}\right\|$ is tempered. On the other hand, since $z_{j}^{*}, j=1, \cdots, N$ 
are independent Gaussian random variables we have that

$$
\prod_{j=1}^{N} \mathbb{E}\left(\left\|S_{B_{j}}\left(-z_{j}^{*}\right)\right\|\left\|S_{B_{j}}\left(z_{j}^{*}\right)\right\|\right)<\infty
$$

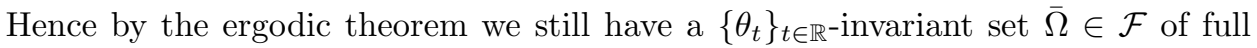
measure such that

$$
\begin{aligned}
\lim _{t \rightarrow \pm \infty} \frac{1}{t} \int_{0}^{t}\left\|T\left(\theta_{\tau} \omega\right)\right\|\left\|T^{-1}\left(\theta_{\tau} \omega\right)\right\| d \tau & =\mathbb{E}\|T\|\left\|T^{-1}\right\| \\
& \leq \prod_{j=1}^{N} \mathbb{E}\left(\left\|S_{B_{j}}\left(-z_{j}^{*}\right)\right\|\left\|S_{B_{j}}\left(z_{j}^{*}\right)\right\|\right) .
\end{aligned}
$$

According to Remark 4.2 we can change our metric dynamical system with respect to $\bar{\Omega}$. However the new metric dynamical system will be denoted by the old symbols $(\Omega, \mathcal{F}, \mathbb{P}, \theta)$.

We formulate an evolution equation with random coefficients but without white noise

$$
\frac{d \psi}{d t}=\left(A+\sum_{j=1}^{N} \lambda_{j} z_{j}^{*}\left(\theta_{t} \omega\right) B_{j}\right) \psi+T^{-1}\left(\theta_{t} \omega\right) f\left(T\left(\theta_{t} \omega\right) \psi\right)
$$

and initial condition $\psi(0)=x \in H$.

Lemma 4.3. Suppose that $A, B_{1}, \cdots, B_{N}$ satisfy the preceding assumptions. Then i) the random evolution equation (26) possesses a unique solution, and this solution generates a random dynamical system.

ii) if $\psi$ is the random dynamical system in i),

$$
\phi(t, \omega, x)=T\left(\theta_{t} \omega\right) \psi\left(t, \omega, T^{-1}(\omega) x\right)
$$

is another random dynamical system for which the process

$$
(\omega, t) \rightarrow \phi(t, \omega, x)
$$

solves (20) for any initial condition $x \in H$.

Proof. i) We mention only that by the continuity of $t \rightarrow T\left(\theta_{t} \omega\right)$ the mapping $x \rightarrow T^{-1}\left(\theta_{t} \omega\right) f\left(T\left(\theta_{t} \omega\right) x\right)$ is Lipschitz continuous, where the Lipschitz constant is uniformly bounded for $t \in[0, T], T>0$. Hence we can prove the existence and uniqueness of a mild solution of this equation for any $\omega$. The proof of measurability is straightforward.

ii) According to the chain rule (and omitting for simplicity the arguments in the 
mappings $T$ and $\psi$ we have

$$
\begin{aligned}
d(T \psi)= & \sum_{i=1}^{N} B_{i} T \psi \circ d z_{i}^{*}+T\left(A+\sum_{i=1}^{N} \lambda_{i} z_{i}^{*} B_{i}\right) \psi d t+f(T \psi) d t \\
= & -\sum_{i=1}^{N} \lambda_{i} B_{i} z_{i}^{*} T \psi d t+\sum_{i=1}^{N} B_{i} T \psi \circ d w_{i} \\
& +A T \psi d t+\sum_{i=1}^{N} \lambda_{i} B_{i} z_{i}^{*} T \psi d t+f(T \psi) d t \\
= & A \phi+\sum_{i=1}^{N} B_{i} \phi \circ d w_{i}+f(\phi)
\end{aligned}
$$

since $T$ commutes with $A, B_{i}$. Hence, the mapping defined by (27) is a solution of (20). On account of Lemma 2.2, $\phi$ and $\psi$ are conjugated random dynamical systems.

Now we can formulate the first main conclusion of this section.

Theorem 4.4. Suppose $A, B_{1}, \cdots, B_{N}$ mutually commute and $W=\left(w_{1}, \cdots, w_{N}\right)$ satisfies the assumptions at the beginning of this section. In addition, suppose that

$$
a+\sum_{j=1}^{N} b_{j} \lambda_{j} \mathbb{E}\left|z_{j}^{*}\right|+L_{f} \prod_{j=1}^{N} \mathbb{E}\left(\left\|S_{B_{j}}\left(-z_{j}^{*}\right)\left|\left\|\mid S_{B_{j}}\left(z_{j}^{*}\right)\right\|\right)<0 .\right.\right.
$$

Then the random dynamical system generated by (20) admits a unique exponentially stable stationary solution given by a random fixed point $X^{*}$ in the sense of random dynamical systems.

Proof. 1) Suppose that the random dynamical system $\psi$ has the random fixed point $Y^{*}$. Then by $(27)$

$$
X^{*}(\omega)=T(\omega) Y^{*}(\omega)
$$

is a random fixed point of $\phi$. If

$$
\psi(t, \omega, x)-Y^{*}\left(\theta_{t} \omega\right)
$$

tends to zero for $t \rightarrow \infty$ exponentially fast, then

$$
\phi(t, \omega, x)-X^{*}\left(\theta_{t} \omega\right)
$$

also tends to zero for $t \rightarrow \infty$ exponentially fast since the norm of the linear mapping $T(\omega)$ is tempered. Hence to prove the theorem it is sufficient to show that (26) has a unique exponentially attracting fixed point.

2) In order to obtain a random fixed point for $\psi$ we first check the condition for Theorem 2.4.

According to the Lipschitz continuity of $f$ we have

$$
\|f(x)\| \leq L_{f}\|x\|+\|f(0)\| .
$$

This inequality gives us the differential inequality

$$
\begin{aligned}
\frac{d\|\psi(t)\|^{2}}{d t} \leq & 2\left(a+\sum_{j=1}^{N} \lambda_{j} b_{j}\left|z_{j}^{*}\left(\theta_{t} \omega\right)\right|+\left\|T\left(\theta_{t} \omega\right)\right\|\left\|T^{-1}\left(\theta_{t} \omega\right)\right\| L_{f}+\frac{\varepsilon}{2}\right)\|\psi(t)\|^{2} \\
& +\frac{1}{\varepsilon}\left\|T^{-1}\left(\theta_{t} \omega\right)\right\|^{2}\|f(0)\|^{2} .
\end{aligned}
$$


for a sufficiently small $\varepsilon>0$. Comparing the coefficients of (29) we have $\|\psi(t)\|^{2} \leq$ $R^{2}(t)$ if $\|\psi(0)\|^{2} \leq R^{2}(0)$ where $R^{2}(t)$ is the solution of

$$
\begin{aligned}
\frac{d R^{2}}{d t}= & 2\left(a+\sum_{j=1}^{N} \lambda_{j} b_{j}\left|z_{j}^{*}\left(\theta_{t} \omega\right)\right|+\left\|T\left(\theta_{t} \omega\right)\right\|\left\|T^{-1}\left(\theta_{t} \omega\right)\right\| L_{f}+\frac{\varepsilon}{2}\right) R^{2} \\
& +\frac{1}{\varepsilon}\left\|T^{-1}\left(\theta_{t} \omega\right)\right\|^{2}\|f(0)\|^{2}
\end{aligned}
$$

Under the assumptions of the theorem, this equation has the unique exponentially stable stationary solution $t \rightarrow R^{2}\left(\theta_{t} \omega\right)$ defined by the random variable

$$
\begin{aligned}
R^{2}(\omega): & =\int_{-\infty}^{0} \frac{1}{\varepsilon}\left\|T^{-1}\left(\theta_{t} \omega\right)\right\|^{2}\|f(0)\|^{2} \times \\
& \times \exp \left(\int_{t}^{0} 2\left(a+\sum_{j=1}^{N} \lambda_{j} b_{j}\left|z_{j}^{*}\left(\theta_{\tau} \omega\right)\right|+\left\|T\left(\theta_{\tau} \omega\right)\right\|\left\|T^{-1}\left(\theta_{\tau} \omega\right)\right\| L_{f}+\frac{\varepsilon}{2}\right) d \tau\right) d t
\end{aligned}
$$

for $\omega \in \Omega$, which in turn follows easily by the variation of constants formula. This random variable is tempered (and finite), see Lemma 4.6 below. In particular the ball in $H$ given by $G(\omega):=B(0,2 R(\omega))$ is mapped into itself:

$$
\psi(t, \omega, G(\omega)) \subset G\left(\theta_{t} \omega\right) \quad \text { for } \omega \in \Omega
$$

Indeed this ball is tempered. In addition, this ball has the property (11). To see this we consider the differential equation (30) with some random tempered initial condition $r^{2}(\omega)$. If we replace $\omega$ by $\theta_{-t} \omega$ for the solution of (30) at time $t$ we have by the variation of constants formula

$$
\begin{aligned}
& r^{2}\left(\theta_{-t} \omega\right) \exp \left(\int_{t}^{0} 2\left(a+\sum_{j=1}^{N} \lambda_{j} b_{j}\left|z_{j}^{*}\left(\theta_{\tau} \omega\right)\right|+\left\|T\left(\theta_{\tau} \omega\right)\right\|\left\|T^{-1}\left(\theta_{\tau} \omega\right)\right\| L_{f}+\frac{\varepsilon}{2}\right) d \tau\right) \\
& \quad+\int_{t}^{0} \frac{1}{\varepsilon}\left\|T^{-1}\left(\theta_{s} \omega\right)\right\|^{2}\|f(0)\|^{2} \times \\
& \quad \times \exp \left(\int_{s}^{0} 2\left(a+\sum_{j=1}^{N} \lambda_{j} b_{j}\left|z_{j}^{*}\left(\theta_{\tau} \omega\right)\right|+\left\|T\left(\theta_{\tau} \omega\right)\right\|\left\|T^{-1}\left(\theta_{\tau} \omega\right)\right\| L_{f}+\frac{\varepsilon}{2}\right) d \tau\right) d s .
\end{aligned}
$$

This term tends to $R^{2}(\omega)$ as $t \rightarrow-\infty$ which follows by Birkhoff's ergodic theorem, (23), (28), (25). In particular the first term tends to zero. From Theorem 2.4 we just obtain the existence of a random attractor $\mathcal{A}=\{\mathcal{A}(\omega)\}_{\omega \in \Omega} \subset G$.

Set

$$
\Delta \psi\left(t, \omega, x_{1}, x_{2}\right)=\psi\left(t, \omega, x_{1}\right)-\psi\left(t, \omega, x_{2}\right) .
$$

Then we have

$$
\frac{d\|\Delta \psi(t)\|^{2}}{d t} \leq 2\left(a+\sum_{j=1}^{N} \lambda_{j} b_{j}\left|z_{j}^{*}\left(\theta_{t} \omega\right)\right|+\left\|T\left(\theta_{t} \omega\right)\right\|\left\|T^{-1}\left(\theta_{t} \omega\right)\right\| L_{f}\right)\|\Delta \psi(t)\|^{2} .
$$


We can conclude by the invariance property (9)

$$
\begin{aligned}
\sup _{y_{1}, y_{2} \in A(\omega)} & \left\|y_{1}-y_{2}\right\|^{2} \leq \sup _{x_{1}, x_{2} \in A\left(\theta_{-t} \omega\right)}\left\|x_{1}-x_{2}\right\|^{2} \times \\
& \times \exp \left(\int_{t}^{0} 2\left(a+\sum_{j=1}^{N} \lambda_{j} b_{j}\left|z_{j}^{*}\left(\theta_{\tau} \omega\right)\right|+\left\|T\left(\theta_{\tau} \omega\right)\right\|\left\|T^{-1}\left(\theta_{\tau} \omega\right)\right\| L_{f}\right) d \tau\right)
\end{aligned}
$$

Since $\mathcal{A} \subset G$, it is tempered, and we obtain from the properties of $z_{j}^{*}$ and $T$ that the right hand side tends to zero for $\omega \in \Omega$, hence $\mathcal{A}(\omega)$ is a random fixed point denoted by $Y^{*}$. To see that $Y^{*}$ is exponentially attracting we note that

$$
\begin{aligned}
& \sup _{x \in D(\omega)}\left\|\psi(t, \omega, x)-Y^{*}\left(\theta_{t} \omega\right)\right\|^{2}=\sup _{x \in D(\omega)}\left\|\psi(t, \omega, x)-\psi\left(t, \omega, Y^{*}(\omega)\right)\right\|^{2} \\
& \leq \sup _{x \in D(\omega)}\left\|x-Y^{*}(\omega)\right\|^{2} \times \\
& \quad \times \exp \left(\int_{0}^{t} 2\left(a+\sum_{j=1}^{N} \lambda_{j} b_{j}\left|z_{j}^{*}\left(\theta_{\tau} \omega\right)\right|+\left\|T\left(\theta_{\tau} \omega\right)\right\|\left\|T^{-1}\left(\theta_{\tau} \omega\right)\right\| L_{f}\right) d \tau\right)
\end{aligned}
$$

and the right hand side tends to zero exponentially fast for $\omega \in \Omega$.

Since we now know that $\mathcal{A}(\omega)$ is a single point we have similarly to (32) and (10) that

Corollary 4.5. The random fixed point $Y^{*}$ (hence $X^{*}$ ) attracts tempered random sets in the pullback sense.

We now prove the temperedness of $R^{2}$.

Lemma 4.6. For $\omega \in \Omega$ the mapping $t \rightarrow R^{2}\left(\theta_{t} \omega\right)$ has subexponential growth, hence $R^{2}$ is tempered.

Proof. We abbreviate

$$
\begin{aligned}
& \alpha(\omega)=a+\sum_{j=1}^{N} \lambda_{j} b_{j}\left|z_{j}^{*}(\omega)\right|+\|T(\omega)\|\left\|T^{-1}(\omega)\right\| L_{f}+\frac{\varepsilon}{2}, \quad \mathbb{E} \alpha=: \bar{\alpha}<0, \\
& \beta(\omega)=\frac{1}{\varepsilon}\left\|T^{-1}(\omega)\right\|^{2}\|f(0)\|^{2} .
\end{aligned}
$$

By the definition of $\Omega$ we have

$$
\int_{t}^{0} \alpha\left(\theta_{\tau} \omega\right) d \tau \sim \bar{\alpha}|t| \quad \text { for } \omega \in \Omega, \quad t \rightarrow-\infty .
$$

In addition $t \rightarrow \beta\left(\theta_{t} \omega\right)$ has sub-exponential growth for $t \rightarrow \pm \infty$ such that

$$
R^{2}(\omega)=\int_{-\infty}^{0} \exp \left(\int_{t}^{0} \alpha\left(\theta_{\tau} \omega\right)\right) \beta\left(\theta_{t} \omega\right) d t<\infty
$$


For an arbitrary $c>0,0<\varepsilon<\min \left(-\bar{\alpha}, \frac{c}{2}\right) / 4$ and negative $s<s_{0}(\omega, \varepsilon)$ we then have

$$
\begin{aligned}
& e^{c s} \int_{-\infty}^{0} e^{\int_{t}^{0} \alpha\left(\theta_{\tau+s} \omega\right) d \tau} \beta\left(\theta_{s+t} \omega\right) d t \\
& \quad \leq e^{\frac{c}{2} s} \int_{-\infty}^{0} e^{\int_{t+s}^{0}\left(\alpha\left(\theta_{\tau} \omega\right)-\bar{\alpha}\right) d \tau-\int_{s}^{0}\left(\alpha\left(\theta_{\tau} \omega\right)-\bar{\alpha}\right) d \tau-\bar{\alpha} t+s \frac{c}{2}+\log ^{+} \beta\left(\theta_{t+s} \omega\right)} d t \\
& \quad \leq e^{\frac{c}{2} s} \int_{-\infty}^{0} e^{-3 \varepsilon(t+s)-\bar{\alpha} t+\frac{c}{2} s} d t \leq e^{\frac{c}{2} s} \int_{-\infty}^{0} e^{\varepsilon t} d t .
\end{aligned}
$$

But the right hand side tends to zero for $s \rightarrow-\infty$.

Similarly, we obtain the convergence for $t \rightarrow+\infty$, see also Arnold [1] Proposition 4.1.3.

Corollary 4.7. Suppose that $f$ and $B_{j}$ commute, i.e. it holds

$$
T(\omega)^{-1} f(T(\omega) x)=f(x), \text { for } \omega \in \Omega, x \in H .
$$

Then the conclusion of Theorem 4.4 also holds if instead of (28) we assume

$$
a+L_{f}<0 .
$$

Proof. Since the expectation of $z_{j}^{*}$ depends on $\lambda_{j}$ such that

$$
\mathbb{E}\left|z_{j}^{*}\right| \leq \frac{1}{\sqrt{\lambda_{j}}}
$$

we can choose $\lambda_{j}$ sufficiently small so that $\sum_{j=1}^{N} b_{j} \lambda_{j} \mathbb{E}\left|z_{j}^{*}\right|$ is also arbitrarily small.

Remark 4.8. Usually $B_{j}$ and $f$ commute if they are diagonal in some orthogonal basis of $H$ (see Kwiecinska [18] and our example in Section 6) .

The commutativity assumptions for $B_{i}$ ensure that (20) can be transformed into a random differential equation. However, there exist other transformations for very special classes of stochastic evolution equations to be transformed into random differential equations, see e.g. Flandoli and Lisei [11] and Mohammed et al. [21]. For the resulting random evolution equation our theory can be applied.

\section{Non-Linear perturbations}

We now want to show that if we change the nonlinear part of (3) continuously, then the fixed points also change continuously. For this we study the family of problems indexed by $n \in \mathbb{Z}^{+}$given by

$$
d X=A X d t+f_{n}(X) d t+B(X) d W, \quad X(0)=u_{0} .
$$

We suppose that the constants $\mu_{n}$ given by (13) corresponding to the functions $f_{n}$, satisfy $\mu_{n}<0$ uniformly for $n \in \mathbb{Z}^{+}$. We will also denote by $\Phi_{n}\left(\cdot, 0, u_{0}\right), n=$ $0,1,2, \ldots$, the solution to (33), and by $X_{n}^{*}$ their associated random fixed points. Then, our objective is to prove that the $X_{n}^{*}$ are close to $X_{0}^{*}$ if the $f_{n}$ is close to $f_{0}$ in some sense. 
Theorem 5.1. Consider the family of stochastic evolution equations (33). Suppose that

$$
\mu:=\sup _{n \in \mathbb{Z}^{+}}\left(2 a+2 L_{f_{n}}+L_{B}\right)<0,
$$

and, in addition, that

$$
\lim _{n \rightarrow \infty} f_{n}(u)=f_{0}(u), \quad \text { for } u \in H .
$$

Then, for the corresponding fixed points we have

$$
\left(\mathrm{L}_{2}\right) \lim _{n \rightarrow \infty} X_{n}^{*}=X_{0}^{*} \text {. }
$$

Proof. One can find the idea of the proof in Zeidler [26] Proposition 1.2 . Denoting $\|\cdot\|_{L_{2}}^{2}=\mathbb{E}\|\cdot\|^{2}$ and taking into account (12), we have

$$
\begin{aligned}
& \left\|X_{n}^{*}-X_{0}^{*}\right\|_{L_{2}}=\left\|\Phi_{n}\left(1,-1, X_{n}^{*}\left(\theta_{-1} \cdot\right)\right)-\Phi_{0}\left(1,-1, X_{0}^{*}\left(\theta_{-1} \cdot\right)\right)\right\|_{L_{2}} \\
& \quad=\left\|\Phi_{n}\left(1,0, X_{n}^{*}\right)-\Phi_{0}\left(1,0, X_{0}^{*}\right)\right\|_{L_{2}} \\
& \quad \leq\left\|\Phi_{n}\left(1,0, X_{n}^{*}\right)-\Phi_{n}\left(1,0, X_{0}^{*}\right)\right\|_{L_{2}}+\left\|\Phi_{n}\left(1,0, X_{0}^{*}\right)-\Phi_{0}\left(1,0, X_{0}^{*}\right)\right\|_{L_{2}} \\
& \quad \leq e^{\mu}\left\|X_{n}^{*}-X_{0}^{*}\right\|_{L_{2}}+\left\|\Phi_{n}\left(1,0, X_{0}^{*}\right)-\Phi_{0}\left(1,0, X_{0}^{*}\right)\right\|_{L_{2}},
\end{aligned}
$$

so

$$
\left\|X_{n}^{*}-X_{0}^{*}\right\|_{L_{2}} \leq \frac{1}{1-e^{\mu}}\left\|\Phi_{n}\left(1,0, X_{0}^{*}\right)-\Phi_{0}\left(1,0, X_{0}^{*}\right)\right\|_{L_{2}} .
$$

Now it is not hard to prove that the right hand side tends to zero. Indeed, we set $X_{n}(t)=\Phi_{n}\left(t, 0, X_{0}^{*}\right), n \in \mathbb{Z}^{+}$, corresponding to the solution of (3) with $f=f_{n}$. Then by the Ito formula we obtain

$$
\begin{gathered}
\frac{d}{d t} \mathbb{E}\left\|X_{n}(t)-X_{0}(t)\right\|^{2} \leq \mu \mathbb{E}\left\|X_{n}(t)-X_{0}(t)\right\|^{2}+\mathbb{E} \| f_{n}\left(X_{0}(t)-f_{0}\left(X_{0}(t)\right) \|^{2}\right. \\
\mathbb{E}\left\|X_{n}(0)-X_{0}(0)\right\|^{2}=0 .
\end{gathered}
$$

The inequality

$$
\left\|f_{n}\left(X_{0}(t)\right)\right\|^{2} \leq 2 \sup _{n \in \mathbb{Z}^{+}}\left\|f_{n}(0)\right\|^{2}+2 L\left\|X_{0}(t)\right\|^{2}, \quad L:=\sup _{n \in \mathbb{Z}^{+}} L_{f_{n}}<\infty
$$

allows us to find an integrable majorant for $\left\|f_{n}\left(X_{0}(t)\right)-f_{0}\left(X_{0}(t)\right)\right\|^{2}$ such that pointwise convergence of $f_{n}(u)$ to $f_{0}(u)$ and the variation of constants formula yield the convergence for the right hand side of (34).

We now consider a family of evolution equations (20) with $f=f_{n}$. To obtain a family of equations of the form (26) we can apply the transformation $T$ which is independent of $n$.

Theorem 5.2. Suppose that the Lipschitz constants of $f_{n}$ are uniformly bounded by $L$, that

$$
a+\sum_{j=1}^{N} b_{j} \lambda_{j} \mathbb{E}\left|z_{j}^{*}\right|+L \Pi_{j=1}^{N} \mathbb{E}\left(\left\|S_{B_{j}}\left(-z_{j}^{*}\right)\right\|\left\|S_{B_{j}}\left(z_{j}^{*}\right)\right\|\right)<0
$$

and that

$$
\lim _{n \rightarrow \infty} f_{n}(x)=f_{0}(x) \quad \text { for } x \in H .
$$

Let $X_{n}^{*}, n \in \mathbb{Z}^{+}$, be the random fixed points of (20) with $f_{n}$ instead of $f$. Then

$$
\lim _{n \rightarrow \infty} X_{n}^{*}(\omega)=X_{0}^{*}(\omega), \quad \text { for } \omega \in \Omega \text {. }
$$


Proof. As in the proof of Theorem 4.4 we investigate the random dynamical systems $\psi_{n}$ generated by (26) with $f=f_{n}$ and fixed points $Y_{n}^{*}(\omega)$ attracting tempered sets, see Corollary 4.5. We have $\sup _{n \in \mathbb{N}}\left\|f_{n}(0)\right\|<\infty$, so, as in the proof of Theorem 4.4, there exists a tempered set $G(\omega)$ containing all fixed points $Y_{n}^{*}(\omega)$. This set is given by a ball $B(0,2 R(\omega))$ where $R^{2}$ is a stationary solution of the one-dimensional affine differential equation (30) where $L_{f}$ has to be replace by $L=\sup _{n \in \mathbb{Z}^{+}} L_{f_{n}}$ and $\|f(0)\|$ by $\sup _{n \in \mathbb{Z}^{+}}\left\|f_{n}(0)\right\|$. By the assumptions $R^{2}(\omega)$ exists.

We have

$$
\begin{aligned}
\left\|Y_{n}^{*}(\omega)-Y_{0}^{*}(\omega)\right\| & =\left\|\psi_{n}\left(t, \theta_{-t} \omega, Y_{n}^{*}\left(\theta_{-t} \omega\right)\right)-\psi_{0}\left(t, \theta_{-t} \omega, Y_{0}^{*}\left(\theta_{-t} \omega\right)\right)\right\| \\
& \leq\left\|\psi_{n}\left(t, \theta_{-t} \omega, Y_{n}^{*}\left(\theta_{-t} \omega\right)\right)-\psi_{n}\left(t, \theta_{-t} \omega, Y_{0}^{*}\left(\theta_{-t} \omega\right)\right)\right\| \\
& +\left\|\psi_{n}\left(t, \theta_{-t} \omega, Y_{0}^{*}\left(\theta_{-t} \omega\right)\right)-\psi_{0}\left(t, \theta_{-t} \omega, Y_{0}^{*}\left(\theta_{-t} \omega\right)\right)\right\| .
\end{aligned}
$$

Since the second factor on the right hand side of (32) is independent of $n$ if we replace $L_{f}$ by $L$, and $Y_{0}^{*}, Y_{n}^{*}$ are contained in the tempered ball $G$, we have for any $\varepsilon>0, \omega \in \Omega$ and $t$ that the first term on the right hand side is less than $\varepsilon / 2$. In addition, for this $t$ similar to the proof of Theorem 5.1 the second term on the right hand side is also less than $\varepsilon / 2$ if $n$ is large. We obtain $\left\|Y_{n}^{*}(\omega)-Y_{0}^{*}(\omega)\right\|<\varepsilon$ for large $n$. Since the transformation $T$ is a homeomorphism we have the conclusion.

\section{An EXAmple}

Let us take $H=L^{2}(\mathcal{O})$ and $V=H_{0}^{1}(\mathcal{O})$ where $\mathcal{O}$ is a bounded domain in $\mathbb{R}^{d}$ with a smooth boundary. Consider the Laplace operator $A=\Delta$ with the Dirichlet boundary condition. Theorem IX.31 in Brezis [2] ensures the existence of a sequence of real numbers $\left\{\nu_{n}\right\}_{n \geq 1}$ such that $0<\nu_{1}<\nu_{2}<\cdots<\nu_{n}<\cdots$, and $\nu_{n} \rightarrow+\infty$ (namely, the eigenvalues of the Laplacian), and a sequence $\left\{e_{n}\right\}_{n \geq 1} \subset V \cap C^{\infty}(\mathcal{O})$ of associated eigenvectors (i.e. $-\Delta e_{n}=\nu_{n} e_{n}$ on $\mathcal{O}$ ) which is a complete orthonormal basis in $H$.

On the other hand, let us consider operators $B_{i}, i=1, \cdots, N$ such that they are diagonalizable in the same basis and are bounded from above and below (see Kwiecinska [18]), i.e., there exist constants $d_{i}^{k}, k=1, \ldots, N$ such that

$$
\left(B_{k} e_{i}, e_{j}\right)=d_{i}^{k} \delta_{i j}, \quad k=1, \ldots, N ; \quad i, j=1,2, \ldots
$$

The assumption that the operators $B_{k}$ are bounded from above and below is equivalent to the condition that there exist positive constants $m_{k}, M_{k}$ such that

$$
0<m_{k} \leq\left|d_{i}^{k}\right| \leq M_{k}, \quad k=1, \ldots, N ; \quad i=1,2, \ldots
$$

Under these conditions the operators $B_{i}$ generate $C_{0}$-groups $S_{B_{i}}(t)=e^{B_{i} t}$.

Finally, consider a Lipschitz continuous function $f$ from $H$ into $H$ defined as $f(u)(x)=F(u(x)), x \in \mathcal{O}$ with $F: \mathbb{R} \rightarrow \mathbb{R}$ Lipschitz continuous with constant $L_{f}$. Recall that $\langle A u, u\rangle \leq-\nu_{1}\|u\|^{2}$ for all $u \in V$, and therefore the semigroup generated by $A$ satisfies

$$
\|S(t)\| \leq e^{-\nu_{1} t} \quad \text { for all } \quad t \geq 0
$$

We now study the stochastic evolution equation

$$
d X=(A X+f(X)) d t+\sum_{i=1}^{N} B_{i} X d w_{i} .
$$


It follows that the constant $\mu$ in Theorem 3.3 satisfies

$$
\mu=-2 \nu_{1}+2 L_{f}+L_{B} \leq-2 \nu_{1}+2 L_{f}+\sum_{i=1}^{N} M_{i}^{2} .
$$

If $\mu<0$, Theorem 3.3 implies the existence of a unique stationary solution to our problem which is exponentially stable in mean square. Also, thanks to Lemma 3.1 ii) and Theorem 3.5 the almost sure exponential stability of this stationary solution holds.

Observe that $\mu$ is negative only if the Lipschitz constants $L_{f}$ and $L_{B}$ are sufficiently small. However, by using the technique of random dynamical systems we can prove stability behaviour even for larger values of the constant $L_{B}$.

Indeed, we will be able to apply Theorem 4.4. To this end, we consider our equation (35) in its Stratonovich equivalent form

$$
d X=\left[\left(A-\frac{1}{2} \sum_{i=1}^{N} B_{i}^{2}\right) X+f(X)\right] d t+\sum_{i=1}^{N} B_{i} X \circ d w_{i} .
$$

Denote $C=A-\frac{1}{2} \sum_{i=1}^{N} B_{i}^{2}$ which also generates a $C_{0}$-semigroup $S_{C}(t)$. If the operators $A, B_{1}, \ldots, B_{N}$ commute mutually, then this semigroup $S_{C}(t)$ is given as

$$
S_{C}(t)=S(t) e^{-\frac{t}{2} \sum_{i=1}^{N} B_{i}^{2}} .
$$

Now, by easy computations (see [18]) we can deduce

$$
e^{-\frac{t}{2} \sum_{i=1}^{N} M_{i}^{2}} \leq\left\|e^{-\frac{t}{2} \sum_{i=1}^{N} B_{i}^{2}}\right\| \leq e^{-\frac{t}{2} \sum_{i=1}^{N} m_{i}^{2}}
$$

and assumption (28) in Theorem 4.4 becomes

$$
\widetilde{\mu}=-\nu_{1}-\frac{1}{2} \sum_{i=1}^{N} m_{i}^{2}+\sum_{i=1}^{N} M_{i} \lambda_{i} \mathbb{E}\left|z_{i}^{*}\right|+L_{f} \prod_{i=1}^{N} \mathbb{E}\left(\left\|S_{B_{i}}\left(-z_{i}^{*}\right)\right\|\left\|S_{B_{i}}\left(z_{i}^{*}\right)\right\|\right)<0 .
$$

Consequently, if the operators $B_{i}$ are such that $\widetilde{\mu}<0$, we can apply Theorem 4.4 and ensure that there exists a unique exponentially stable stationary solution given by a random fixed point.

Notice that this can provide better stability results than the ones obtained in Section 3. Indeed, assume for instance that $B_{i} u=m_{i} u$ where $m_{i} \in \mathbb{R}^{+}$for $i, \cdots, N$. This means that $m_{i}=M_{i}$, and it is easy to check that

$$
\widetilde{\mu}=-\nu_{1}-\frac{1}{2} \sum_{i=1}^{N} m_{i}^{2}+\sum_{i=1}^{N} m_{i} \lambda_{i} \mathbb{E}\left|z_{i}^{*}\right|+L_{f},
$$

since now $\left\|S_{B_{i}}\left(-z_{i}^{*}\right)\right\|\left\|S_{B_{i}}\left(z_{i}^{*}\right)\right\|=1$. Then, if

$$
-\nu_{1}-\frac{1}{2} \sum_{i=1}^{N} m_{i}^{2}+L_{f}<0
$$

(what happens if, for example, the noise intensities $m_{i}$ are large enough), we can choose stationary process $z_{i}^{*}$ corresponding to $\lambda_{i}$ such that $\widetilde{\mu}<0$. Thus, some kind of stabilization has been obtained for the non-trivial stationary solution.

Acknowledgements. This work was partially supported by the DAAD (Germany), and the Ministerio de Ciencia y Tecnología (Spain) under the projects HA2001-0075 and BFM2002-03068. 
T. Caraballo would like to thank B. Schmalfuss and his family for the kind hospitality they all offered him during his visit in November 2002.

\section{REFERENCES}

[1] L. Arnold. Random Dynamical Systems. Springer, New York, 1998.

[2] H. Brezis. Analyse Fonctionnelle. Masson, Paris, 1983.

[3] T. Caraballo and J. Langa. Comparison of the long-time behavior of linear Ito and Stratonovich partial differential equations. Stochastic Analysis and Applications, 19(2):183195, 2001.

[4] T. Caraballo, K. Liu, and X. Mao. On stabilization of partial differential equations by noise. Nagoya Mathematical Journal, 161:155-170, 2001.

[5] T. Caraballo and J. Real. On the pathwise exponential stability of nonlinear stochastic partial differential equations. Stochastic Analysis and Applications, 12(5):517-525, 1994.

[6] C. Castaing and M. Valadier. Convex Analysis and Measurable Multifunctions. LNM 580. Springer-Verlag, Berlin-Heidelberg-New York, 1977.

[7] P.L. Chow. Stability of nonlinear stochastic-evolution equations. Journal of Mathematical Analysis and Applications, 89(2):400-419, 1982.

[8] H. Crauel, A. Debussche, and F. Flandoli. Random attractors. Journal of Dynamics and Differential Equations, 9:307-341, 1997.

[9] R. Dautray and J.L. Lions. Analyse mathématique et calcul numérique pour les sciences et les techniques, volume 1 of Collection du Commissariat à l'Énergie Atomique: Série Scientifique. Masson, Paris, 1984.

[10] G. Da Prato and J. Zabczyk. Stochastic Equations in Infinite Dimensions. University Press, Cambridge, 1992.

[11] F. Flandoli and H. Lisei. Stationary conjugation of flows for parbolic spdes with multiplicative noise and some applications. Manuscript, 2002.

[12] F. Flandoli and B. Schmalfuß. Random attractors for the stochastic 3-D Navier-Stokes equation with multiplicative white noise. Stochastics and Stochastics Reports, 59:21-45, 1996.

[13] W. Grecksch and C. Tudor. Stochastic Evolution Equations-a Hilbert space approach, volume 85 of Mathematical Research. Akademie Verlag, Berlin, 1995.

[14] R. Z. Hasmínskiǔ. Stochastic stability of differential equations. Sijthoff \& Nordhoff, Alphen aan den Rijn, The Netherlands; Rockville, Maryland, USA, 1980.

[15] U.G. Haussmann. Asymptotic stability of the linear Itô equation in infinite dimensions. Journal of Mathematical Analysis and Applications, 65(1):219-235, 1978.

[16] A. Ichikawa. Stability of semilinear stochastic evolution equations. Journal of Mathematical Analysis and Applications, 90(1):12-44, 1982.

[17] N.V. Krylov and B.L. Rozovskii. Ob evoljucionnych stochastičeskich uravneniach, volume 14 of Sovremennych problemy matematiki, pages 71-146. Moskva, 1979.

[18] A.A. Kwiecinska. Stabilization of evolution equations by noise. Proceedings of the American Mathematical Society, 130(10):3067-3074, 2001.

[19] K. Liu and X. Mao. Exponential stability of non-linear stochastic evolution equations. Stochastic Processes and their Applications, 78(2):173-193, 1998.

[20] X. Mao. Exponential Stability of Stochastic Differential Equations. Marcel Dekker, New York, 1994.

[21] S-E. A. Mohammed, T. Zhang, and H. Zhao. The stable manifold theorem for semilinear stochastic evolution equations and stochastic partial differential equations. Preprint, 2003.

[22] B. Øksendal. Stochastic Differential Equations. Springer-Verlag, Berlin-Heidelberg-New York, third edition, 1992.

[23] B. Schmalfuß. Backward cocycles and attractors of stochastic differential equations. In V. Reitmann, T. Riedrich, and N. Koksch, editors, International Seminar on Applied Mathematics-Nonlinear Dynamics: Attractor Approximation and Global Behaviour, pages 185-192, 1992.

[24] B. Schmalfuß. Lyapunov functions and non-trivial stationary solutions of stochastic differential equations. Dynamical Systems, 19(4):303-317, 2001.

[25] R. Temam. Infinite-Dimensional Dynamical Systems in Mechanics and Physics. SpringerVerlag, Berlin-Heidelberg-New York, second edition, 1997. 
[26] E. Zeidler. Nonlinear Functional Analysis and its Applications, volume I. Springer-Verlag, New York, 1985.

E-mail address, Tomás Caraballo: caraball@us.es

E-mail address, Peter E. Kloeden: kloeden@math.uni-frankfurt.de

E-mail address, Björn Schmalfuß: bjoern.schmalfuss@in.fh- merseburg.de

(Tomás Caraballo) Dpto. Ecuaciones Diferenciales y AnÁlisis Numérico, Universidad de Sevilla, Apdo. de Correos 1160, 41080-Sevilla (Spain)

(Peter E. Kloeden) Fachbereich Mathematik, Johann Wolfgang Goethe-Universität, D60054 Frankfurt am Main, Germany

(Björn Schmalfuß) Department of Applied Sciences, University of Technology and Applied Sciences, Geusaer Strasse, D-06217 Merseburg, Germany, 